\title{
Pandemic and the Dynamics of SEIR Model: Case COVID-19
}

\author{
Md. Kamrujjaman ${ }^{1,4}$, Uttam Ghosh ${ }^{* 2}$, and Md. Shahidul Islam ${ }^{3}$ \\ ${ }^{1,3}$ Department of Mathematics, University of Dhaka, Dhaka 1000, Bangladesh \\ ${ }^{2}$ Department of Applied Mathematics, University of Calcutta, Kolkata, India \\ ${ }^{4}$ Department of Mathematics and Statistics, University of Calgary, Calgary, AB, Canada \\ ${ }^{1,4}$ E-mail: kamrujjaman@du.ac.bd \\ ${ }^{2}$ E-mail: uttam_math@yahoo.co.in \\ ${ }^{3}$ E-mail: mshahidul11@yahoo.com
}

\begin{abstract}
:
Rapidly spreading disease, COVID-19 is classified as the human-to-human transmissionable disease and currently is a pandemic in the Globe. In this paper, we propose conceptual mathematical models for COVID-19 outbreak and it's control measurement; quarantine, hospitalization and the effect of panic and anxiety. In this situation, mathematical models are a important tool to employ an effective strategy in order to fight against this pandemic. We establish the positivity and boundedness of solutions, local and global stability analysis of equilibria to examine its epidemiological relevance. To validate the model and estimating the important model parameters and prediction about the disease, we consider the real cases of Italy from $15^{\text {th }}$ Feb to $13^{\text {th }}$ April 2020. In a series of graphical map, we have presented the comparative study to estimate the current scenarios and to predict the control measurement time boundary of the outbreak.
\end{abstract}

Keywords: Coronavirus; SEIR model; stability analysis; COVID-19; numerical analysis. AMS Subject Classification 2010: 37N25, 49J15, 92D30.

\section{Introduction}

Throughout human history, several times pandemics and epidemics have ravaged humanity, oftentimes resulting in massive change in the course of history and end of civilizations. But for the current coronavirus pandemic, the Glove is now facing with a dangerous and destructive phenomenon which crucially threatening the mankind [1, 2]. First it was identified in Wuhan city, Hubei Province of China on December 31, 2019. The World Health

\footnotetext{
${ }^{*}$ Corresponding author: Uttam Ghosh (email: uttam_math@yahoo.co.in)
} 
Organization (WHO) declared the disease as a pandemic and was named SARS-CoV-2 virus (March 11, 2020) [2]. Scientifically it is proven that COVID-19 is an infectious disease which causes respiratory syndrome and transmissible from human-to-human [2]. At this stage, more than 210 countries and territories have reported to have coronavirus patients and increasing the infections exponentially $[2,3]$. The coronavirus is a zoonotic disease, where the primary host was animals and transmitted to humans [4]. The patients faces more critical illness, primarily who has other diseases like diabetes, heart disease, asthma etc. [2].

Till April 20, 2020, according to WHO COVID-19 infected more than 2.3 million people and the total death crosses 0.16 million due to the infection $[1,2]$. Geometrically the number of new reported cases is growing and the dynamics of growth is satisfied several mathematical growth curves which are meaningful to predict the situation of such an outbreak $[3,5,6]$. Due to the severity, as a protection highly infected counties and territories has lockdown and their administrations including WHO are encouraging, advising and even enforcing (some territories) people to stay at home to protect their citizens. The coronavirus pandemic has already started showing immense negative impact on world politics, socio-economics, education and other important global aspects [7, 8]. Moreover, the state of medical emergency is becoming more and more gruesome by every passing day. Therefore, it is an emergency to formulate a mathematical model that effectively describes the development and transmission of the disease to help policymakers make important decisions based on the effective assumptions given by the model. Lack of early measures and ineffective decisions have already been attributed to the massive scale destruction that the pandemic is causing, so we cannot afford to be any more ignorant in this matter. In mathematical modelling, some recent studies provided different guidelines introducing basic reproduction number, Education and Socio-Economic Index and Lockdown strategies, (see $[9,10,12,13]$ and references therein).

In epidemiology, mathematical modelling is widely used to predict the results of an epidemic successfully. Most commonly used epidemic models are SIS, SIR and SEIR models. The Kermack-Mckendric SIR model is a very well established model and used widely for various epidemics [9]. In many cases, there are no visible symptoms infected individuals such as chicken pox, tuberculosis etc. and in that cases an SEIR model is mostly used [10]. Therefore, the model with multiple compartments is the useful tool to predict the nature of recent most dangerous disease, COVID-19.

The main findings in this study are outlined in the following lists:

1. We analyze the stability of the equilibria of the model using the basic reproduction number to understand the severity.

2. Theoretical results are established using local and global analysis of the model.

3. During these periods of Quarantine, we have studied the human behaviors like panic, anxiety and tensions which is increasing the death rate of individuals.

4. Numerical illustration ensures the theoretical results to control the spread of COVID19. 


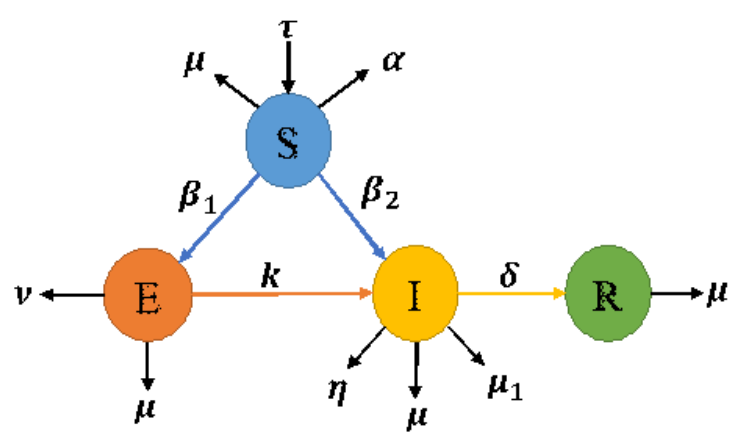

Figure 1: The schematic Diagram of the proposed model

The paper is organized as follows: Mathematical Model is elaborately discussed in section 2. Positivity and boundedness of solution including auxiliary results are described in subsection 2.1. Local-global stability analysis and bifurcation analysis are prescribed in section 3. Section 4 is accomplished the data analysis in comparison with model solution with further prediction to control the epidemic, as a case study in Italy. Finally, section 5 outlines the summary and discussion of the results.

In the following section, we will discuss our mathematical model and formulation of the model elaborately.

\section{$2 \quad$ Model formulation}

Our aim is to develop an epidemic model which is simple but relevant enough to produce effective results upon analysis. The spread of the infection starts with the introduction of a small group of infected individuals to a large population. The population $(N)$ is then divided into four classes; the susceptible $(S)$, the exposed and asymptotic $(E)$, the infected $(I)$ and the recovered $(R)$ at any time $t \geq 0$. Since as per advisory of WHO [2] the infected persons who shows symptom will be isolated in the hospital and hence they will infect only the medical persons but the exposed \& asymptotic class is interacting with the common people and will spread disease. Unlike the classical SIR model, there is an extra class in our model namely the exposed \& asymptotic (E) because in case who are also spreading the diaseas [14]. To include the above defined facts we consider the rate of infection the form $\beta_{1} E S+\beta_{2} I S$. The flow diagram of proposed COVID-19 disease dynamics model is shown in Figure 1 and corresponding proposed model formulated as given in equation (2.1): 
Table 1: Model parameters and their descriptions

\begin{tabular}{llll}
\hline Notation & Interpretations & Notation & Interpretations \\
\hline$\tau$ & Recruitment rate of S class & $k$ & Infected rate of E class \\
$\mu$ & Natural death rate & $\gamma$ & Panic/tension/anxiety of E class \\
$\alpha$ & Panic/tension/anxiety of S class & $\delta$ & Recovery rate of I class \\
$\beta_{1}$ & Transmission rate of infection from E class & $\mu_{1}$ & Death rate due to infection \\
$\beta_{2}$ & Transmission rate of infection from I class & $\eta$ & Panic/tension/anxiety of I class \\
\hline
\end{tabular}

$$
\left\{\begin{array}{l}
\frac{d S}{d t}=\tau-(\mu+\alpha) S-\left(\beta_{1} E+\beta_{2} I\right) S \\
\frac{d E}{d t}=\left(\beta_{1} E+\beta_{2} I\right) S-(k+\mu+\gamma) E \\
\frac{d I}{d t}=k E-\left(\mu+\mu_{1}+\delta+\eta\right) I \\
\frac{d R}{d t}=\delta I-\mu R
\end{array}\right.
$$

with the initial conditions $S(0)>0, E(0) \geq 0, I(0)>0, R(0) \geq 0$, where the interpretation of parameters is presented in Table 1.

\subsection{Positivity and boundedness of solutions}

An important feature of an epidemiological relevant model is the positivity and boundedness of the solutions. Therefore, it is important to prove that all the variables are nonnegative for all time $t \geq 0$ which implies that any solution that has positive initial values will remain positive for all time $t \geq 0$.

Theorem 1. The closed region $\Omega=\left\{(S, E, I, R) \in \mathbb{R}^{4}+: 0<N \leq \frac{\tau}{\mu}\right\}$ is positively invariant set for the system (2.1).

Proof. Let $N(t)=S(t)+E(t)+Q(t)+R(t)$ then

$$
\frac{d N}{d t}=\frac{d S}{d t}+\frac{d E}{d t}+\frac{d Q}{d t}+\frac{d R}{d t}
$$

So, from model (2.1):

$$
\frac{d N}{d t} \leq \tau-\mu N-\left(\mu_{1}+\eta\right) I-\alpha S-\gamma E
$$

which yields

$$
\frac{d N}{d t} \leq \tau-\mu N
$$


It implies that $\frac{d N}{d t}<0$ whenever $N(t)>\frac{\tau}{\mu}$. Thus, the right-hand side of equation (2.2) implies that $\frac{d N}{d t}$ is bounded by $\tau-\mu$.

Integrating the inequality (2.3), using initial condition, we obtain

$$
N(t) \leq N(0) e^{-\mu t}+\frac{\tau}{\mu}\left[1-e^{-\mu t}\right] .
$$

Letting $t$ tends to infinity, asymptotically we get $N(t) \leq \frac{\tau}{\mu}$.

Therefore, $\Omega$ is positively invariant of the model (2.1) so that no solution path leaves through any boundary of $\Omega$. This proves that the formulated model is relevant both mathematically and epidemiologically.

The model is considered in the biologically feasible region. We need to show that any phase trajectory that started anywhere in the non-negative region $\mathbb{R}^{4}$ of the phase space eventually enters the feasible region $\Omega$ and remains in $\Omega$ thereafter. It can be done by proving that $\Omega$ is a positively invariant set and global attractor of the system.

\subsection{Basic reproduction number, DFE and EEP}

The basic reproduction number is an important threshold condition in the analysis of an infectious disease. It determines whether the disease will die out or persist in the population as time increases. It is defined to be the number of secondary infections produced by one primary infection in a population where everyone is susceptible and is denoted by $R_{0}$. If $R_{0}>1$, one primary infection can produce more than one secondary infection. This implies that the disease-free equilibrium (DFE) is unstable. As a result, an epidemic breaks out. If $R_{0}<1$, the situation is thought to be under control. In this case, the disease-free equilibrium (DFE) will be locally asymptotically stable and the disease cannot persist in the population. So, when a pandemic breaks out, an effective strategy should be developed so that the reproduction number reduces to less than 1 as soon as possible [12, 14, 15].

Since the considered model has DEF $E_{0}=\left(\frac{\tau}{\mu+\alpha}, 0,0,0\right)$, hence the basic can be found analytically. Using next generation matrix method [15], the reproduction number for the COVID-19 model given by (2.1) can be calculated from the relation $R_{0}=\rho\left(F V^{-1}\right)$, that is the spectral radius of $F V^{-1}$ [15] where

$$
F=\left[\begin{array}{cc}
\frac{\beta_{1} \tau}{\mu+\alpha} & \frac{\beta_{2} \tau}{\mu+\alpha} \\
0 & 0
\end{array}\right]
$$

and

$$
V=\left[\begin{array}{cc}
k+\mu+\gamma & 0 \\
-k & \mu+\mu_{1}+\delta+\eta
\end{array}\right]
$$

Therefore, the spectral radius of $F V^{-1}$ is

$$
R_{0}=\rho\left(F V^{-1}\right)=\frac{\tau \beta_{1}\left(\mu+\delta+\mu_{1}+\eta\right)+\tau \beta_{2} k}{(\mu+\alpha)(k+\mu+\gamma)\left(\mu+\delta+\mu_{1}+\eta\right)}
$$


To find the endemic equilibrium state of the model we set

$$
\frac{d S}{d t}=0, \frac{d E}{d t}=0, \frac{d I}{d t}=0, \frac{d R}{d t}=0
$$

Solving the above system, we get the epidemic equilibrium (EEF) state

$$
E^{*}=\left(S_{1}, E_{1}, I_{1}, R_{1}\right)
$$

where

$$
\begin{gathered}
S_{1}=\frac{\tau\left(\mu+\delta+\mu_{1}+\eta\right)}{k \beta_{2} E+\left(\mu+\alpha+\beta_{1} E\right)\left(\mu+\delta+\mu_{1}+\eta\right)}, I_{1}=\frac{k E_{1}}{\mu+\eta+\delta+\mu_{1}} \\
E_{1}=\frac{\left(\mu+\delta+\mu_{1}+\eta\right)(\mu+\alpha)\left(R_{0}-1\right)}{\left(\beta_{1}\left(\mu+\delta+\mu_{1}+\eta\right)+k \beta_{2}\right)}, R_{1}=\frac{\delta k E}{\mu\left(\mu+\eta+\delta+\mu_{1}\right)} .
\end{gathered}
$$

It is obvious from the expressions of the $E_{1}$ the EEF will exists only when $R_{0}>1$. For further analysis, the Jacobian matrix of the system (2.1) at any equilibrium point $(S, E, I, R)$ is given by

$$
J=\left(\begin{array}{cccc}
-\mu-\beta_{1} E-\beta_{2} I-\alpha & -\beta_{1} S & -\beta_{2} S & 0 \\
\beta_{1} E+\beta_{2} I & \beta_{1} S-(k+\mu+\gamma) & \beta_{2} S & 0 \\
0 & k & -\left(\mu+\delta+\mu_{1}+\eta\right) & 0 \\
0 & 0 & \delta & -\mu
\end{array}\right)
$$

\section{$3 \quad$ Stability and bifurcation of the equilibrium states}

In this section we shall establish the stability and bifurcation condition if the equilibrium points. In theorem 2, we shall establish nature of the $E_{0}$ and in theorem 3 nature of $E^{*}$.

\subsection{Stability of disease-free equilibrium state $\left(E_{0}\right)$}

Theorem 2. The disease free equilibrium point is locally as well as globally asymptotically stable if $R_{0}<1$ and unstable if $R_{0}>1$.

Proof. The Jacobian of the system at the disease free equilibrium point is

$$
J\left(E_{0}\right)=\left(\begin{array}{cccc}
-\mu-\alpha & -\frac{\beta_{1} \tau}{\mu+\alpha} & -\frac{\beta_{2} \tau}{\mu+\alpha} & 0 \\
0 & \frac{\beta_{1} \tau}{\mu+\alpha}-(k+\mu+\gamma) & \frac{\beta_{2} \tau}{\mu+\alpha} & 0 \\
0 & k & -\left(\mu+\delta+\mu_{1}+\eta\right) & 0 \\
0 & 0 & \delta & -\mu
\end{array}\right)
$$

The characteristic roots of the Jacobian matrix at $J\left(E_{0}\right)$ are $-\mu-\alpha,-\mu,-\left(\mu+\delta+\eta+\eta_{1}\right.$ and $(\mu+k+\gamma)\left(R_{0}-1\right)$. Since first three roots are negative and other will be negative if $R_{0}<1$ and positive if $R_{0}>1$. Therefore, the disease-free equilibrium state $\left(E_{0}\right)$ is locally asymptotically stable if $R_{0}<1$ and unstable if $R_{0}>1$. 
To prove the global stability of the equilibrium point we construct the Lyapunov function as

$$
\begin{aligned}
L & =I+E \\
\frac{d L}{d t} & =\frac{d I}{d t}+\frac{d E}{d t}=S\left(\beta_{1} E+\beta_{2} I\right)-(\mu+\gamma) E-\left(\mu+\mu_{1}+\delta+\eta\right) I \\
& \leq \frac{(k+\mu+\gamma)\left(\mu_{1}+\mu+\delta+\eta\right)}{k} I\left(\frac{\beta_{2} k \tau}{(\mu+\alpha)(k+\mu+\gamma)\left(\mu_{1}+\mu+\delta+\eta\right)}-1\right) \\
& +E\left(\frac{\beta_{1} \tau(k+\mu+\gamma)}{(\mu+\alpha)(k+\mu+\gamma)}\right)-(\mu+\gamma) E \\
& \leq M\left(R_{0}-1\right)-(\mu+\gamma) E
\end{aligned}
$$

where $M=\operatorname{Max}\left\{\frac{(k+\mu+\gamma)\left(\mu_{1}+\mu+\delta+\eta\right)}{k} I,(k+\mu+\gamma) E\right\}$.

Hence if $R_{0}<1$ then $\frac{d L}{d t}<0$ hence by Lassela-Lyapunov [16, 17] the disease free equilibrium points is globally asymptotically stable.

Since when $R_{0}=1$ i.e. $\beta_{2}=\beta_{2}^{[T C]}=\frac{\left((\mu+\alpha)(k+\mu+\gamma)-\tau \beta_{1}\right)\left(\mu+\delta+\mu_{1}+\eta\right)}{k \tau}$ then one of the eigenvalue vanishes and classical eigen method analysis fails. Then we have to use the Sotomayer theorem $[17,18,21]$ to investigate the nature of the disease free equilibrium points. Let $V$ and $W$ be the eigenvector corresponding to the zero eigenvalue of $J\left(E_{0}\right)$ and $\left[J\left(E_{0}\right)\right]^{T}$ respectively then

$$
V=\left(\begin{array}{c}
\frac{-\left(\delta+\eta+\mu+\mu_{1}\right)(\gamma+k+\mu)}{k(\mu+\alpha)} \\
\frac{\mu+\delta+\mu_{1}+\eta}{k} \\
1 \\
\frac{\delta}{\mu}
\end{array}\right) \text { and } W=\left(\begin{array}{c}
0 \\
2 \\
\frac{(\alpha+\mu)(\gamma+k+\mu)-\beta_{1} \tau}{k(\mu+\alpha)} \\
0
\end{array}\right)
$$

Let $F=\left(\begin{array}{c}\tau-(\mu+\alpha) S-\left(\beta_{1} E+\beta_{2} I\right) S \\ \left(\beta_{1} E+\beta_{2} I\right) S-(k+\mu+\gamma) E \\ k E-\left(\mu+\delta+\mu_{1}+\eta\right) I \\ \delta I-\mu R\end{array}\right)$ then

$$
\begin{aligned}
& \left.W^{T} F_{\beta_{2}}\right|_{E_{0}, \beta_{2}=\beta_{2}^{[T C]}}=0, \\
& \left.W^{T} D F_{\beta_{2}}\right|_{E_{0}, \beta_{2}=\beta_{2}[T C]} V=-\frac{\tau\left(\mu+\delta+\mu_{1}+\eta\right)}{k(\mu+\alpha)} \neq 0, \\
& \left.W^{T} D^{2} F_{\beta_{2}}\right|_{E_{0}, \beta_{2}=\beta_{2}^{[T C]}}(V, V)=\frac{-2\left(\mu+\delta+\mu_{1}+\eta\right)^{2}(\gamma+k+\mu) \beta_{1}}{k^{2}(\mu+\alpha)} \neq 0 .
\end{aligned}
$$

Hence the system experiences transcritical bifurcation when the rate of infection by the $E-\operatorname{class}\left(\beta_{2}\right)$ crosses the critical value $\beta_{2}=\beta_{2}^{[T C]}$. Thus to spreading the disease the rate of transmission plays important role. There is critical values of the rate of infection by the $E$ - class below of which the disease easy to control but above of which the society will experience endemic disease spreading. 


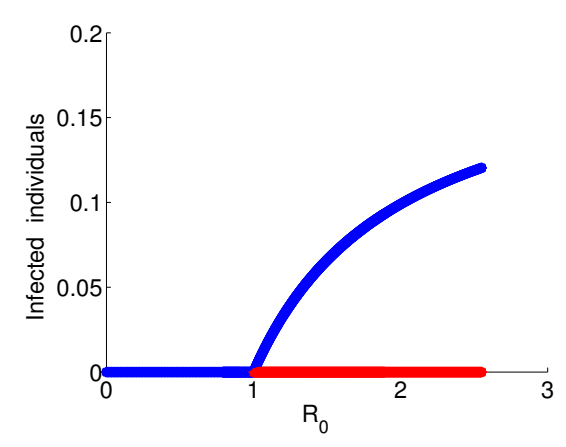

Figure 2: Transcritical bifurcation analysis using the dynamics of $R_{0}$ : The blue line corresponds the stable equilibrium point and the red line corresponds to unstable.

It is clear from the Figure 2 that for the basic reproduction number, $R_{0}<1$, the system (2.1) stable and there is no endemic; disease free equilibrium is stable. While $R_{0}>1$ a stable endemic equilibrium point arises and the DEF become unstable i.e. here exchange of stability of the equilibrium points occurs.

\subsection{Stability of endemic equilibrium state}

Theorem 3. The endemic equilibrium state $E^{*}\left(S_{1}, E_{1}, I_{1}, R_{1}\right)$ is stable if $R_{0}>1$.

Proof. Recall the Jacobian of the system (2.1) at any equilibrium point $(S, E, I, R)$ and we have

$$
J=\left(\begin{array}{cccc}
-\mu-\beta_{1} E-\beta_{2} I-\alpha & -\beta_{1} S & -\beta_{2} S & 0 \\
\beta_{1} E+\beta_{2} I & \beta_{1} S-(k+\mu+\gamma) & \beta_{2} S & 0 \\
0 & k & -\left(\mu+\delta+\mu_{1}+\eta\right) & 0 \\
0 & 0 & \delta & -\mu
\end{array}\right)
$$

At the endemic equilibrium point $E^{*}$, calculating the Jacobian matrix $\mathrm{J}$ and then solving $\operatorname{det}(J-\lambda I)$, the characteristic equation is

$$
(-\mu-\lambda)\left(-\left(\mu+\delta+\mu_{1}+\eta\right)-\lambda\right)\left[\lambda^{2}-a \lambda+b\right]=0
$$

where

$$
\begin{gathered}
a=\beta_{1} S_{1}-k-2 \mu-\alpha-\gamma+\frac{\beta_{2} S_{1} k}{\mu+\delta+\mu_{1}+\eta}, \\
b=\left(\mu+\beta_{1} E+\beta_{2} I\right)(k+\mu+\gamma)+(\alpha+\mu)\left(\beta_{1} S_{1}-k-\mu-\alpha-\gamma+\frac{\beta_{2} S_{1} k}{\mu+\delta+\mu_{1}+\eta}\right) .
\end{gathered}
$$

Since roots of the characteristic equations corresponding to $J\left(E^{*}\right)$ are $\lambda_{1}=-\mu<0$, $\lambda_{2}=-\left(\mu+\delta+\mu_{1}+\eta\right)<0$ and other two satisfies the following quadratic equation

$$
\lambda^{2}-a \lambda+b=0
$$

It is noted that two roots of (3.1) will be negative if $a<0$ and $b>0$. Hence the proof. 
In the following section, we will estimate the parametric values to illustrate the numerical results for further applications.

\section{Parameter estimation, model validation and predic- tion}

The nonlinear system in (2.1) can be solved using the numerical methods and we can observe the dynamics of the model. To numerically solve the data we estimated the parameters first $[1,19,20]$.

\subsection{Case study: Italy}

Using Matlab minimization technique and fmincon software package, we have estimated the important model parameters using the Italy infection cases from $15^{\text {th }}$ Feburary to $13^{\text {th }}$ April 2020 which are given in Table 2. To execute the Matlab package, we have considered the initial population size as

$$
S(0)=60461826, E(0)=150, I(0)=3, \text { and } R(0)=0
$$

where $E(0)$ is estimated and rest of them are collected from [1]. The fitness of the model with the real can be verified computing the residual. The residuals are defined as

$$
\text { residuals }=\left\{Y_{j}-I\left(t_{j}\right) \mid j=1,2,3 \ldots, n\right\}
$$

where $Y_{j}-$ is the $j^{\text {th }}$ day cumulative infection data and $I\left(t_{j}\right)$ - model predicted cumulative infected data of same day. If the residuals are randomly distributed then we can say that the fitness is reasonably good [20].

Table 2: Parameters estimation for Italy.

\begin{tabular}{lll}
\hline Parameter & Value & References \\
\hline$\tau$ & 0.05812 & Estimated \\
$\beta_{1}$ & $6.955360551762161 \times e^{-09}$ & Estimated \\
$\beta_{2}$ & $1.411447332674123 \times e^{-08}$ & Estimated \\
$\mu$ & 0.05 & Estimated \\
$k$ & $1 / 14$ & Estimated \\
$\gamma$ & $0.12-0.25$ & {$[1]$} \\
$\mu_{1}$ & 0.0001 & Estimated \\
$\delta$ & 0.0037 & Estimated \\
$\alpha$ & $0.12-0.25$ & {$[1]$} \\
$\eta$ & $0.15-0.30$ & {$[1]$} \\
\hline
\end{tabular}




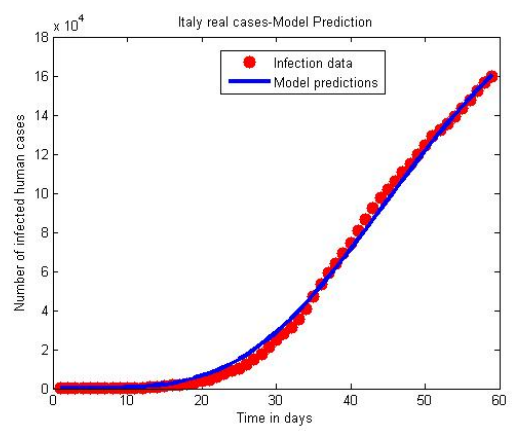

(a)

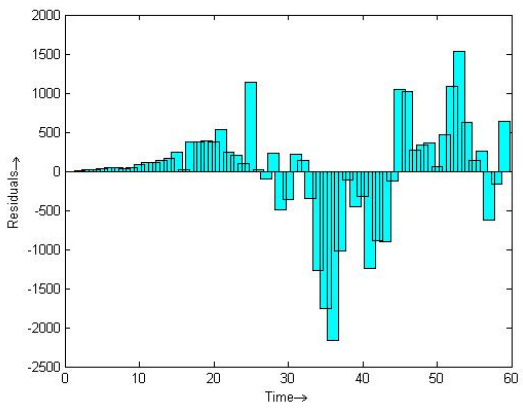

(b)

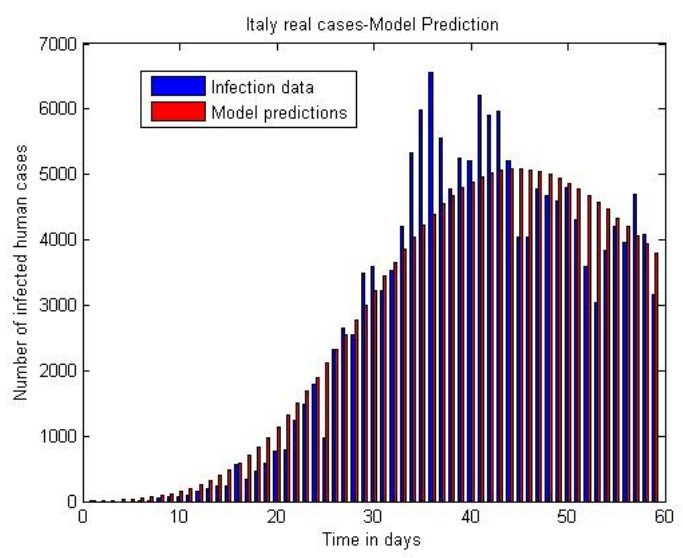

(c)

Figure 3: (a) Fitting model to cumulative cases in Italy, (b) Residuals of the fit, (c) BAR diagram for 59 days.

The estimated model parameters and their sensitivity indices are given in Table 2 . To validate the model we consider the real case of COVID-19 infection of Italy from 15 February to 13 April 2020 i.e. the real cases for 59 days. In Figure 3(a-c) respectively we have presented the best fitted cumulative infected cases and the same of real cases, residuals of the best fitted data and the Bar diagram of the per day new infection of real cases and the model predictions. The randomness of the residual shows that the fitness is best.

The model is fitted to the cumulative number of infected cases, which has been presented in Figure 3(a) and the residuals of the fit is presented in Figure $3(b)$, which shows that the residuals are small and random. The daily infected cases have been presented in Figure 3(c). In Figure 3, the plot is presented the time series of infected population for 59 days. It is clear from the Figure 3 that disease will continue upto $1^{\text {st }}$ week of June, 2020 in Italy if all the conditions remain same with a controllable number of infections. 


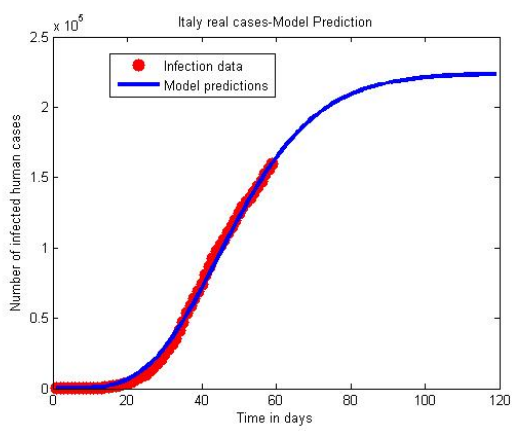

(a)

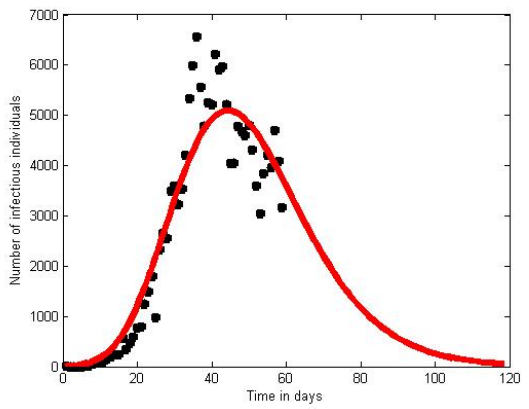

(b)

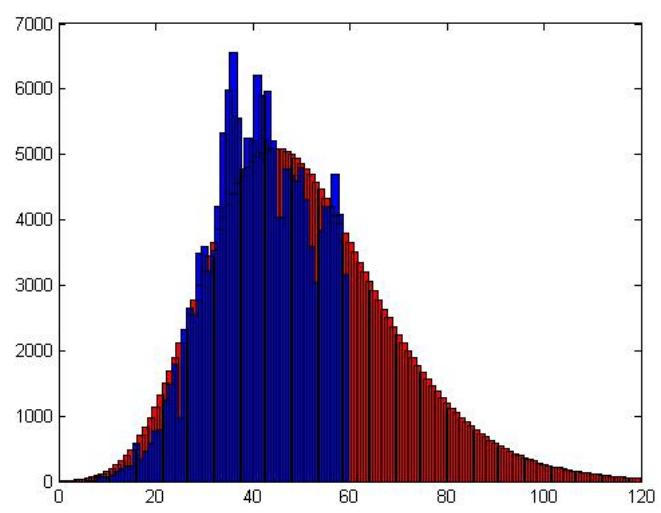

(c)

Figure 4: Prediction in Italy for 119 days (a) Cumulative cases, (b) Per day infection, (c) BAR diagram/day.

In Figure 4(a-c), the diagrams presented the current scenario of infections and we make a prediction with respect to time for cumulative, daily cases and bar diagrams for 119 days. From Figure 4(a), it is seen that the cumulative is flatting after 119 days means there is no additional infection and the disease is under control. It is clear from the Figure 4 that disease will continue upto 2nd week of June, 2020 in Italy if all the conditions remain same with a controllable number of infections; when very few number of daily infected cases will come.

\subsection{Case: World}

At this stage, broadly we are interested to consider all the countries and territories in the world for wide applications and study of theour proposed model.

Now using the parameter values as estimated in Table 3 and initial conditions, we can solve the system by Runge-Kutta 4th order method. Here we have used MATLAB to solve the system in (2.1) and then we generated a graph to analyze the dynamics of susceptible, exposed, infected and recovered population. 
For simplicity, we fix total number of population, $N=1$ such that $N=S+E+I+R=$ 1 , so that each population implies the proportion to the total population.

Table 3: Parameters estimation for World.

\begin{tabular}{lll}
\hline Parameter & Value & References \\
\hline$\tau$ & $5.812 \times 10^{-4}$ & {$[22]$} \\
$\beta_{1}$ & 0.3110 & Estimated \\
$\beta_{2}$ & 0.1555 & Estimated \\
$\mu$ & 0.0005 & {$[22]$} \\
$k$ & 0.1818 & {$[1,8]$} \\
$\gamma$ & {$[0.005-0.05]$} & Estimated \\
$\mu_{1}$ & $9.7 \times 10^{-3}$ & {$[1]$} \\
$\delta$ & 0.009 & Estimated \\
$\alpha$ & {$[0.1-0.2]$} & {$[7]$} \\
$\eta$ & {$[0.05-0.1]$} & {$[7]$} \\
\hline
\end{tabular}

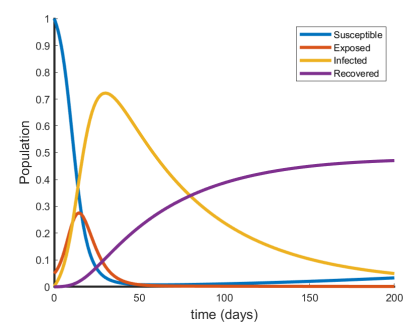

(a)

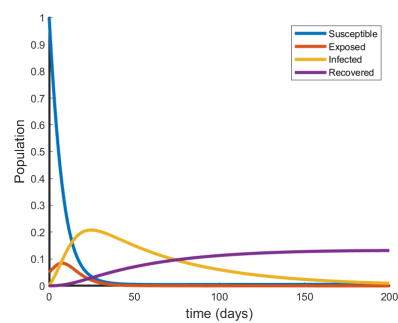

(b)

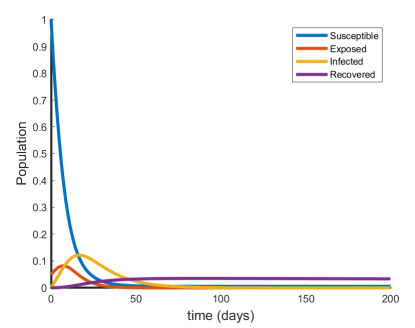

(c)

Figure 5: Dynamics of compartments population over time (a) All class careless (no tension) (b) S class caring (in tension), and (c) S \& E class caring (in tension).

From Figure 5(a), we observe that the susceptible population is decreasing with time, which means more and more people are getting exposed. Since infected population is increasing, therefore they are getting large number of infected individuals over time which can lead to an outbreak in a very short time. If we analyze the rest of the population dynamics, we see that the infected population grows at a very high rate initially meanwhile the recovered population is not growing as much as infected population which results an outbreak. Using the parameters that are estimated in Table 3, we can determine the value of the basic reproduction number, $R_{0}$ which is greater than 1 , means if the estimated data stays this way it will create an outbreak. Comparing with Figure 5(a), it is seen that infection is decreasing eventually with time in both diagrams of Figure $5(\mathrm{~b}-\mathrm{c})$, respectively due to the effect of parameters $\alpha$ and $\eta$. 


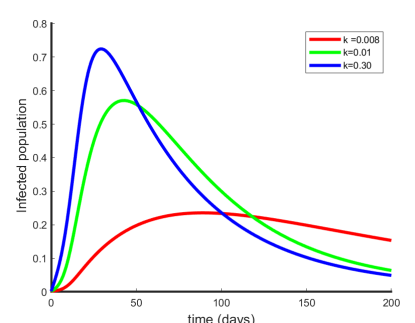

(a)

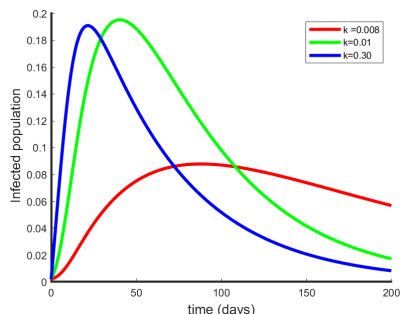

(b)

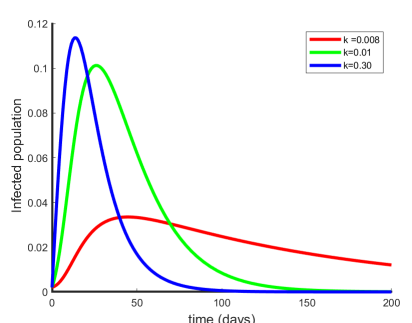

(c)

Figure 6: Dynamics of exposed population infection (a) various infection rates (b) Infection rate while only S class in tension, and (c) Infection rate while both $\mathrm{S} \& \mathrm{E}$ class in tension.

From Figure 6(a), we can say the virus will transmit rapidly over time resulting a vastly infected population and the situation may even get out of control if no necessary steps are taken. In this figure, mainly we focus the significance of parameter, $k$, the infection rate of $\mathrm{E}$ class. If $k$ is increasing then eventually maximum population are being infected. Once again it is observed that infection rate is slowing step by step (see vertical level values) due to the effect of caring (tension) parameters $\alpha$ and $\eta$ (see Figure 6 (b-c) for details) although $k$ is same in all figures. Therefore, it is high time to take precautions to minimize the damage, reduce the value of $k$; socially it means to control public mobility. It is remarked that during numerical illustration, it is found that there is no significant effect parameter $\gamma$ of I class population; may be the reason is we have death rate estimation.

\section{Conclusion}

In this study, we have formulated a SEIR epidemic model for pandemic COVID-19. Considering all major parameters of the progression of the disease, several analytic results established. Theoretically it is proven that the dynamics depends on the basic reproduction number to examine the stability of the system. All the properties necessary for epidemiological relevance have also been proved. We have estimated the parametric values for Italy using the existing real discrete data. The acceptable agreement between data analysis and numerical solutions are established. Finally the inclusion is that to estimate the situation of global pandemic COVID-19, mathematical modelling is an efficient method if the parameters can be estimated properly.

\section{References}

[1] https://www. worldometers.info/coronavirus/

[2] https://www.who.int/emergencies/diseases/novel-coronavirus-2019 
[3] Kamrujjaman M, Mahmud MS and Islam MS. Coronavirus Outbreak and the Mathematical Growth Map of COVID-19. Annual Research \& Review in Biology. 2020; 35(1): $72-78$.

[4] Ahmad T, Khan M, et al. COVID-19: Zoonotic aspects. Travel Medicine and Infectious Disease, ELSEVIER. 2020.

[5] Volpert V, Banerjee M, Petrovskii S. On a Quarantine Model of Coronavirus Infection and Data Analysis. Mathematical Modelling of Natural Phenomena. 2020; 15(24): 16 .

[6] Verhulst PF. Notice sur la loi que la population suit dans son accroissement. Corr. Math. Physics. 1838; 10, 113.

[7] Nathalie V, Valentina L, Rodolphe C, et al. Mathematical modeling of human behaviors during catastrophic events. 4th International Conference on Complex Systems and Applications (ICCSA2014), June 2014, Le Havre, France. 67-74.

[8] Ivorra B, Ramos AM. Application of the Be-CoDiS mathematical model to forecast the international spread of the 2019-20 Wuhan coronavirus outbreak. 2020; DOI: 10.13140/RG.2.2.31460.94081.

[9] Kermack WO and McKendrick AG. A Contribution to the Mathematical Theory of Epidemics. Proceedings of the Royal Society. 1927; 115(772): 700-722.

[10] Castillo-Chavez C and Feng Z. Mathematical models for the disease dynamics of tuberculosis. Advances in Mathematical Population Dynamics-Molecules, Cells and Man. 1996; 1-28.

[11] Chen T, Rui J, Wang Q et al. A mathematical model for simulating the phase-based transmissibility of a novel coronavirus. Infect. Diseases of Poverty, 2020; 9(24), 1-8.

[12] Islam MS, Ira JI, Kabir KMA, Kamrujjaman M. COVID-19 Epidemic Compartments Model and Bangladesh. 2020; preprint, DOI: 10.20944/preprints202004.0193.v1

[13] Mahmud MS, Kamrujjaman M, Jubyrea J, Islam MS. Mathematical Modelling of Social Consciousness to Control the Outbreak of COVID-19. 2020; preprint, DOI: 10.20944/preprints202004.0196.v1

[14] Murray J. Mathematical Biology I, third edition, Springer-Verlag, Heidelberg, 2002.

[15] Diekmann O, Heesterbeek JAP and Roberts MG. The Construction of NextGeneration Matrices for Compartmental Epidemic Models. Journal of The Royal Society Interface. 2009; 7(47): 873-885.

[16] Perko L. Differential Equations and Dynamical Systems. Springer, 2000. 
[17] Martcheva M. An Introduction to Mathematical Epidemiology. Springer, New York, 2015.

[18] DeJesus EX and Kaufman C. Routh-Hurwitz criterion in the examination of eigenvalues of a system of nonlinear ordinary differential equations. American Physical Society. 1987.

[19] Victor AO. Mathematical Predictions for COVID-19 as a Global Pandemic. 2020; medRxiv preprint.

[20] Martcheva M. An Introduction to Mathematical Epidemiology. Springer, New York, 2015.

[21] Lenhart S, Workman JT. Optimal control applied to biological model. Mathematical and compulational biology series. Chapman and Hall/CRC, Boca Raton, 2007.

[22] https://worldpopulationreview.com/

\section{Declarations::}

Ethics approval and consent to participate:

The ethical approval or individual consent was not applicable.

Availability of data and materials:

All data and materials used in this work were publicly available.

Consent for publication:

Not applicable.

Funding:

None.

Acknowledgements:

The author M. Kamrujjaman research was partially supported by TWAS grant: 2019_19169 RG/MATHS/AS_I.

Disclaimer:

None.

Competing Interests:

Authors have declared that no competing interests exist.

Authors' Contributions:

MSI, UG and MK derived the mathematical model, designed the study and the first draft of the manuscript. MK and UG carried out the data analysis, numerical simulations and the parameter estimations. UG, MK and MSI provided the literature review and final drafting. All authors contributed to the reviewing of the manuscript. All authors read and approved the final manuscript. 